\title{
Bromide Causes Facet-Selective Atomic Addition in Gold Nanorod Syntheses
}

\section{Supplemental Information}

Micah Brown ${ }^{1}$ and Benjamin J. Wiley ${ }^{1 *}$

${ }^{1}$ Department of Chemistry, Duke University, 124 Science Drive, Box 90354, Durham, North Carolina 27708, United States.

\section{Contents}

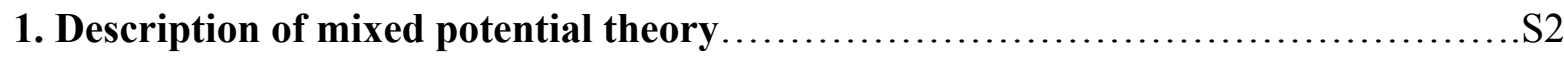

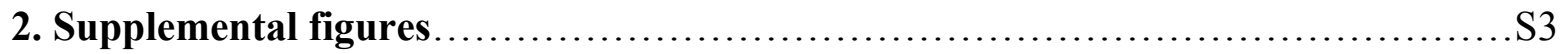

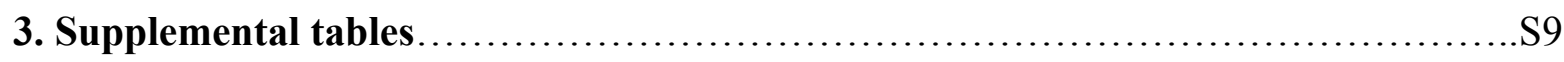

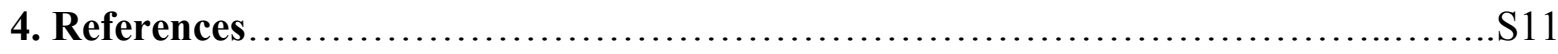




\section{Description of mixed potential theory}

In electroless redox reactions involving the reduction of metal ions (e.g., the conditions of colloidal nanoparticle synthesis described in this work), electrons are spontaneously shuttled from the reducing agent (e.g. AA) to the metal precursor (e.g. Au(I)). For every oxidation event, there is a simultaneous reduction event. Thus, the rates of these two processes are equal, and the associated anodic (oxidation) and cathodic (reduction) current densities are equal and opposite. The ButlerVolmer equation applies to redox processes and describes the net current density as a function of potential. Though typically this equation is applied to a single reversible redox process, it may also be used for paired processes, such as the oxidation of ascorbic acid to dehydroascorbic acid, and the reduction of $\mathrm{Au}(\mathrm{I})$ to elemental $\mathrm{Au}^{0}$ during atomic addition. The Butler-Volmer equation is as follows: ${ }^{1}$

$$
j_{n e t}=j_{o x}+j_{r e d}=j_{0, o x} e^{\left(1-\alpha_{o x}\right) n_{o x} f \eta_{o x}}-j_{0, r e d} e^{-\alpha_{r e d} n_{r e d} f \eta_{r e d}}
$$

Here, $j$ is the current density, $j_{0}$ is the exchange current density, $\alpha$ is the transfer coefficient, $n$ is the number of electrons involved in the reaction, $f$ is equal to $F / R T$, where $F$ is the Faraday constant, $R$ is the ideal gas constant, $T$ is the temperature, and $\eta$ is the overpotential. This equation pertains only to reactions that are kinetically limited and not to those limited by mass transport. At the mixed potential $\left(E_{\mathrm{MP}}\right)$, the anodic and cathodic partial current densities are matched in magnitude $\left(j_{\mathrm{ox}}=-j_{\text {red }}\right)$, and the net current density $\left(j_{\text {net }}\right.$ or $\left.j_{\mathrm{MP}}\right)$ falls to zero. According to mixedpotential theory, $E_{\mathrm{MP}}$ coincides with the potential at which spontaneous, electroless redox reactions take place, as there is neither charge accumulation nor net current flow. The equivalent values of $j_{\text {ox }}$ or $\left|j_{\text {red }}\right|$ at $E_{\mathrm{MP}}$ correspond to the reaction rate (i.e., the rate of Au deposition) and can be extracted from experimentally collected LSV traces using a Tafel plot $(\log |j|$ vs. $E)$. In the high overpotential regions of the Tafel plot (e.g., $\left|E-E_{\mathrm{MP}}\right| \geq 50 \mathrm{mV}$ ), $\log |j|$ depends linearly on $E$. From these regions, Tafel lines may be extrapolated back to $E_{\mathrm{MP}}$ to determine the value of $j_{\text {ox }}$ or $\left|j_{\text {red }}\right|$. The current at $E_{\mathrm{MP}}$ measured in this fashion may be referred to as the mixed potential current $\left(j_{\mathrm{MP}}\right)$.

We note that the mixed potential on $\mathrm{Au}(100)$ is $23 \mathrm{mV}$ lower than the mixed potential on $\mathrm{Au}(111)$. By plotting the currents versus the same potential for $\mathrm{Au}(111)$ and $\mathrm{Au}(100)$, we have implicitly assumed that the two facets are at potentials that differ by $23 \mathrm{mV}$. This assumption may be reasonable given the two facets will have different amounts of adsorbates. The strong correspondence in Figure 2 between the $j_{\text {MP }}$ ratio and the aspect ratio of the nanorods also indicates this assumption may be reasonable. If we do not make this assumption, and instead assume that the two facets are at the same potential, then there will be a charge imbalance in the oxidation and reduction reactions at one or both facets. This charge imbalance in the two half reactions must be compensated for by charge transfer between the facets. As measurement of the amount of charge transfer between facets in a nanoparticle is currently not possible, it is unclear what potential one should assume the two facets to have in this case. Thus, we cannot know or compare what the currents of the half reactions will be on the different facets if we assume the two facets are at the same potential. 


\section{Supplemental figures}
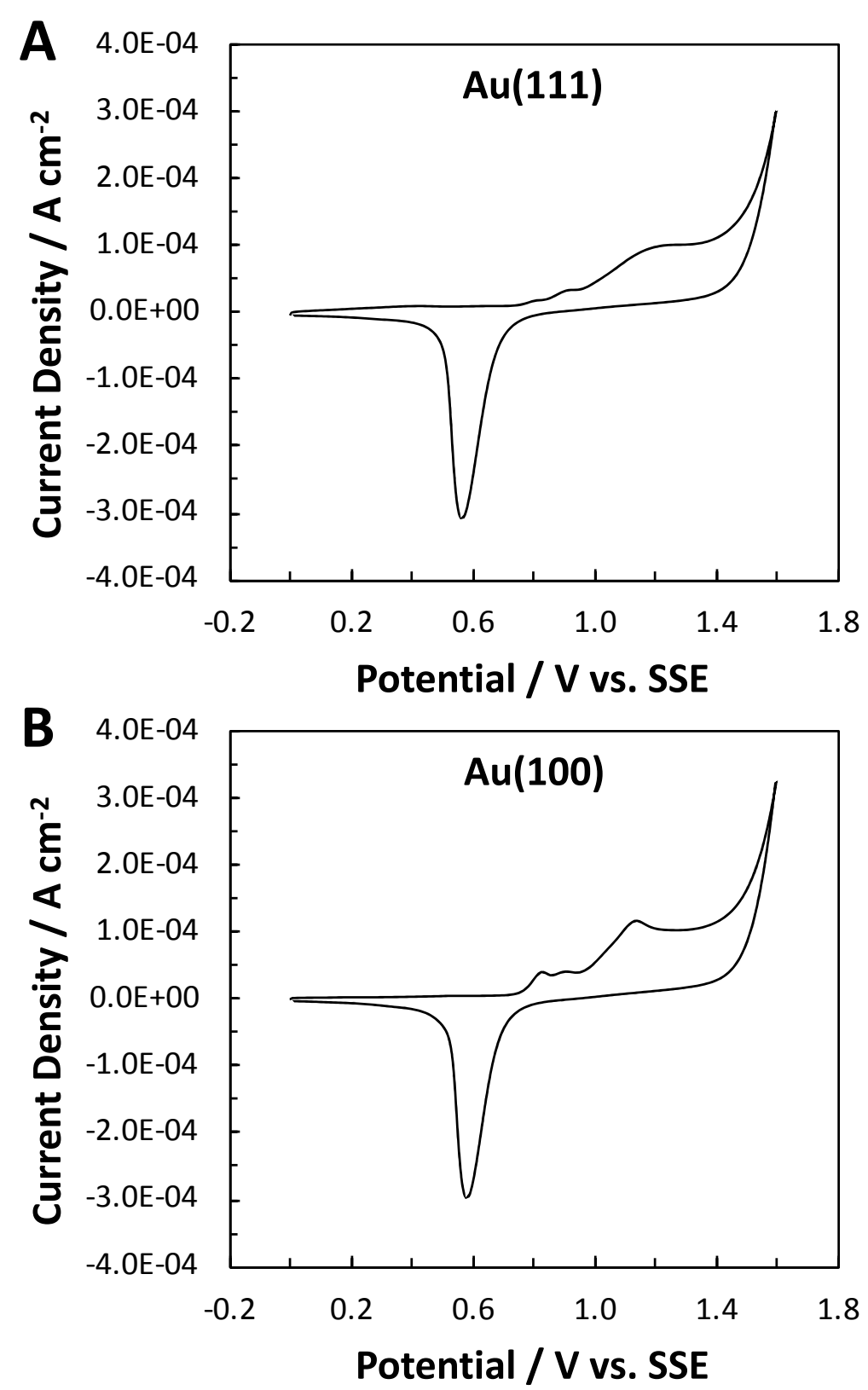

Figure S1. Cyclic voltammograms collected in $0.10 \mathrm{M} \mathrm{HClO}_{4}$ at a scan rate of $20 \mathrm{mV} \mathrm{s}^{-1}$ on (A) $\mathrm{Au}(111)$ and (B) $\mathrm{Au}(100)$ working electrodes immediately after surface preparation. 

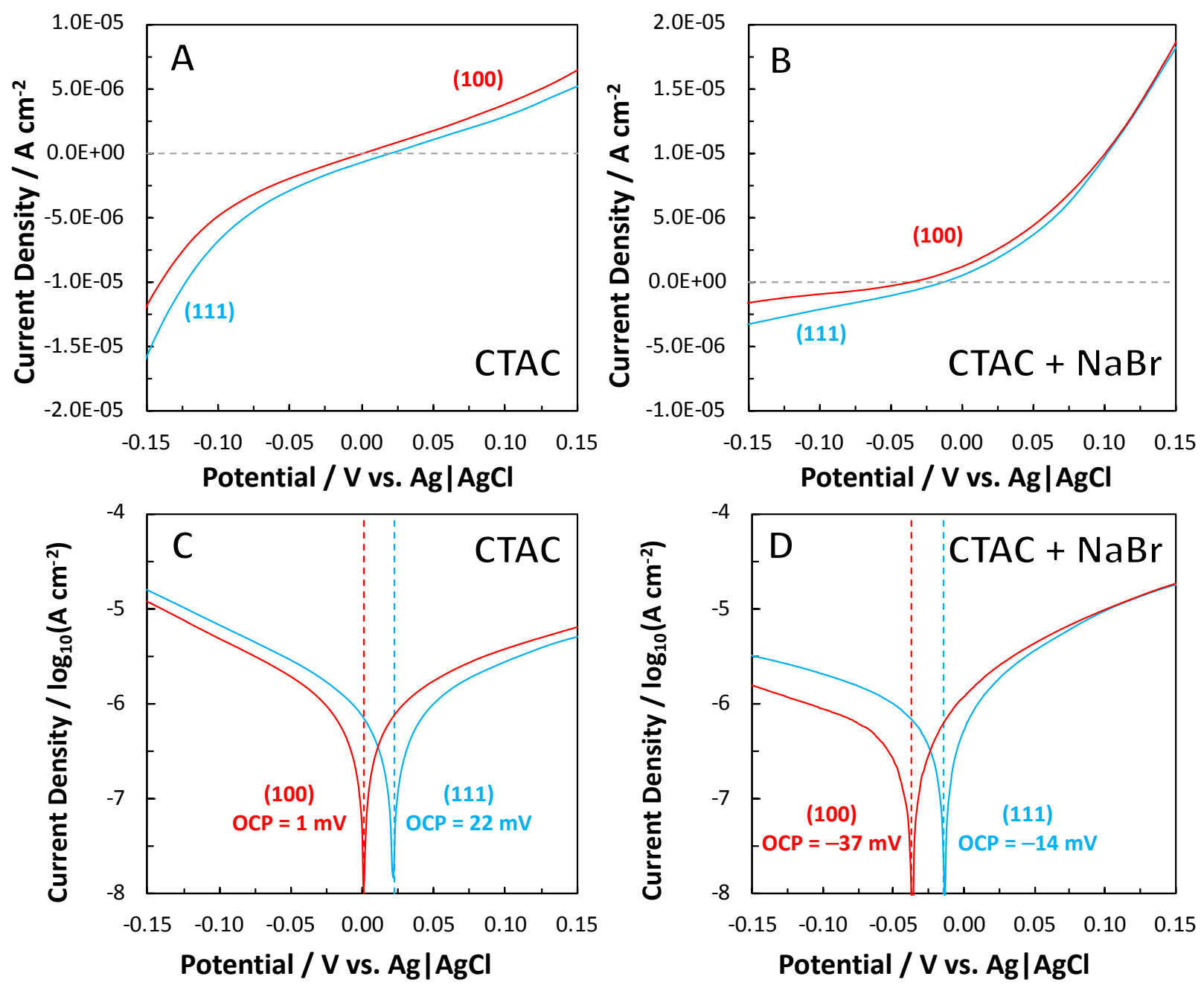

Figure S2. LSVs collected on $\mathrm{Au}(111)$ and $\mathrm{Au}(100)$ single-crystal electrodes in seedless growth solutions containing $0.25 \mathrm{mM} \mathrm{HAuCl}_{4}, 0.55 \mathrm{mM} \mathrm{AA}$, and (A) $50 \mathrm{mM} \mathrm{CTAC}$ or (B) $50 \mathrm{mM} \mathrm{CTAC}$ and $50 \mathrm{mM} \mathrm{NaBr}$. Traces were collected at $25^{\circ} \mathrm{C}$ in $0.2 \mathrm{M} \mathrm{NaF}$ background electrolyte no more than $20 \mathrm{~s}$ after AA injection (scan rate: $1 \mathrm{mV} \mathrm{s}^{-1}$ ). Raw Tafel plots of the traces in (A) and (B) are provided in (C) and (D), respectively. 

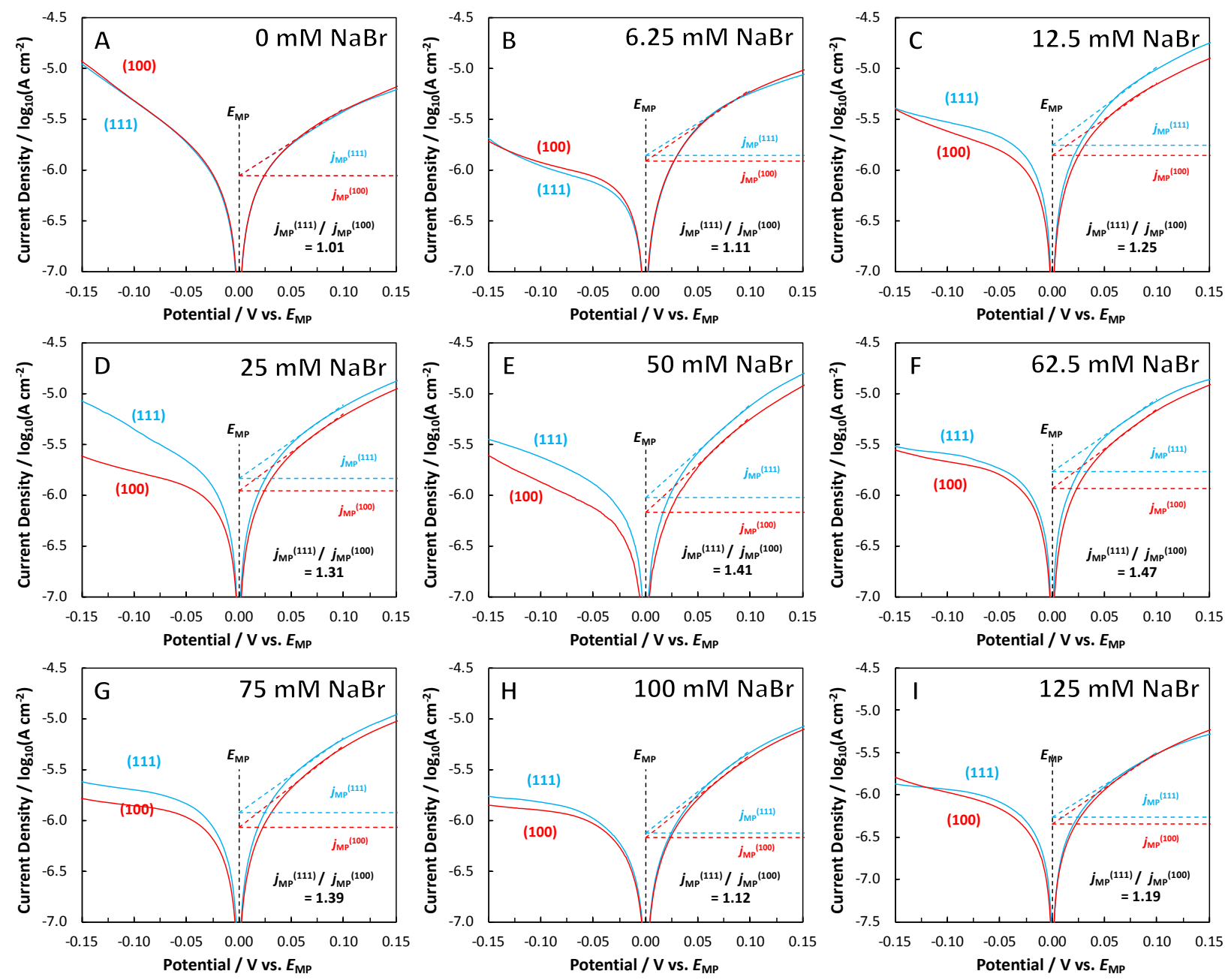

Figure S3. Tafel plots collected on $\mathrm{Au}(111)$ and $\mathrm{Au}(100)$ single-crystal electrodes in seedless growth solutions containing $0.25 \mathrm{mM} \mathrm{HAuCl}_{4}, 0.55 \mathrm{mM} \mathrm{AA}, 50 \mathrm{mM} \mathrm{CTAC}$, and (A) $0 \mathrm{mM}$, (B) $6.25 \mathrm{mM}$, (C) $12.5 \mathrm{mM}$, (D) $25 \mathrm{mM}$, (E) $50 \mathrm{mM}$, (F) $62.5 \mathrm{mM}$, (G) $75 \mathrm{mM}$, (H) $100 \mathrm{mM}$, (I) 125 $\mathrm{mM} \mathrm{NaBr}$. Traces were collected at $25^{\circ} \mathrm{C}$ in $0.2 \mathrm{M} \mathrm{NaF}$ background electrolyte no more than 20 $\mathrm{s}$ after AA injection (scan rate: $1 \mathrm{mV} \mathrm{s}^{-1}$ ). 

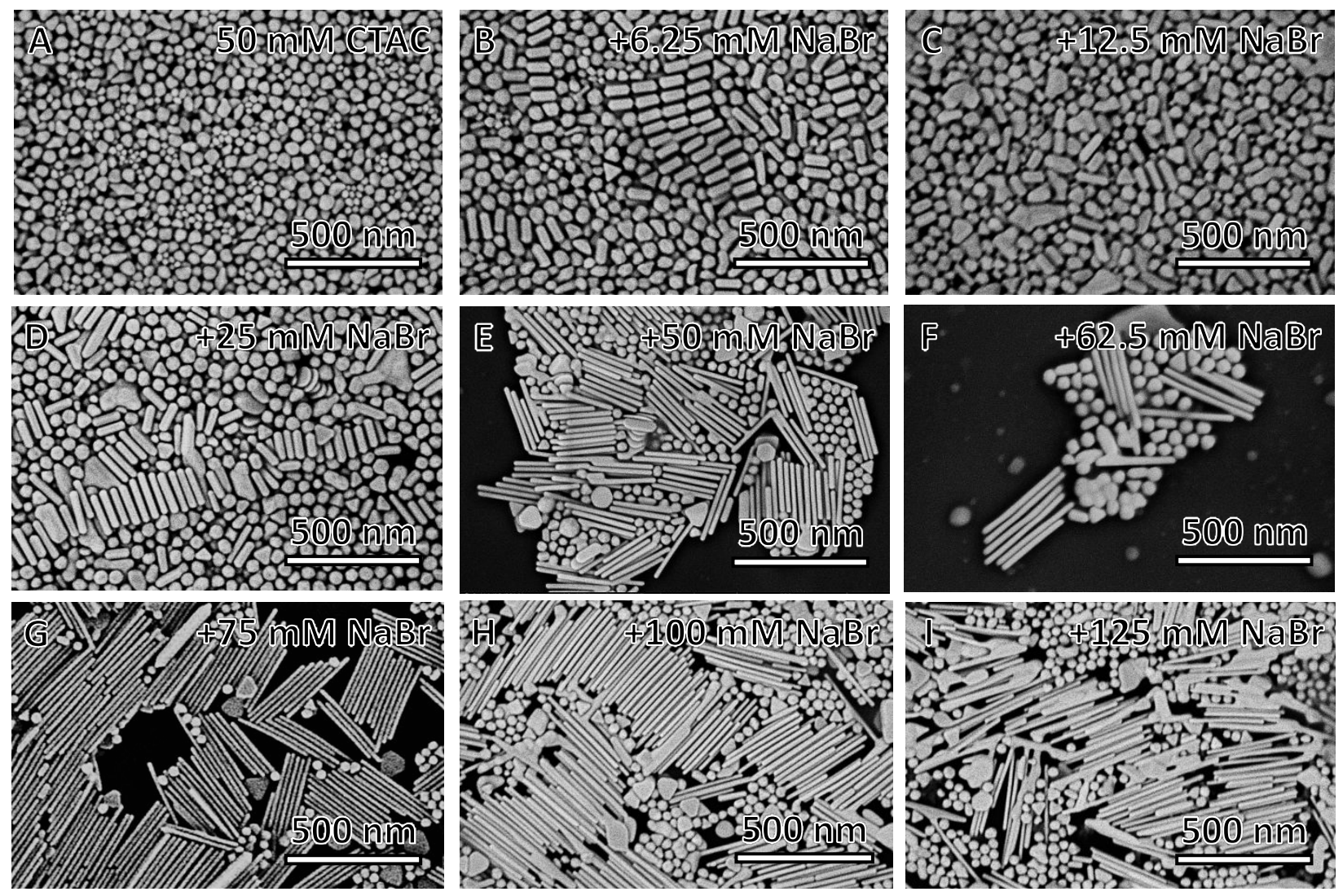

Figure S4. Representative SEM micrographs of the products of various AuNR syntheses from seeded growth solutions containing $0.25 \mathrm{mM} \mathrm{HAuCl}_{4}, 0.55 \mathrm{mM} \mathrm{AA}, 50 \mathrm{mM} \mathrm{CTAC}$, and (A) 0 mM, (B) $6.25 \mathrm{mM}$, (C) $12.5 \mathrm{mM}$, (D) $25 \mathrm{mM}$, (E) $50 \mathrm{mM}$, (F) $62.5 \mathrm{mM}$, (G) $75 \mathrm{mM}$, (H) $100 \mathrm{mM}$, (I) $125 \mathrm{mM} \mathrm{NaBr}$. 

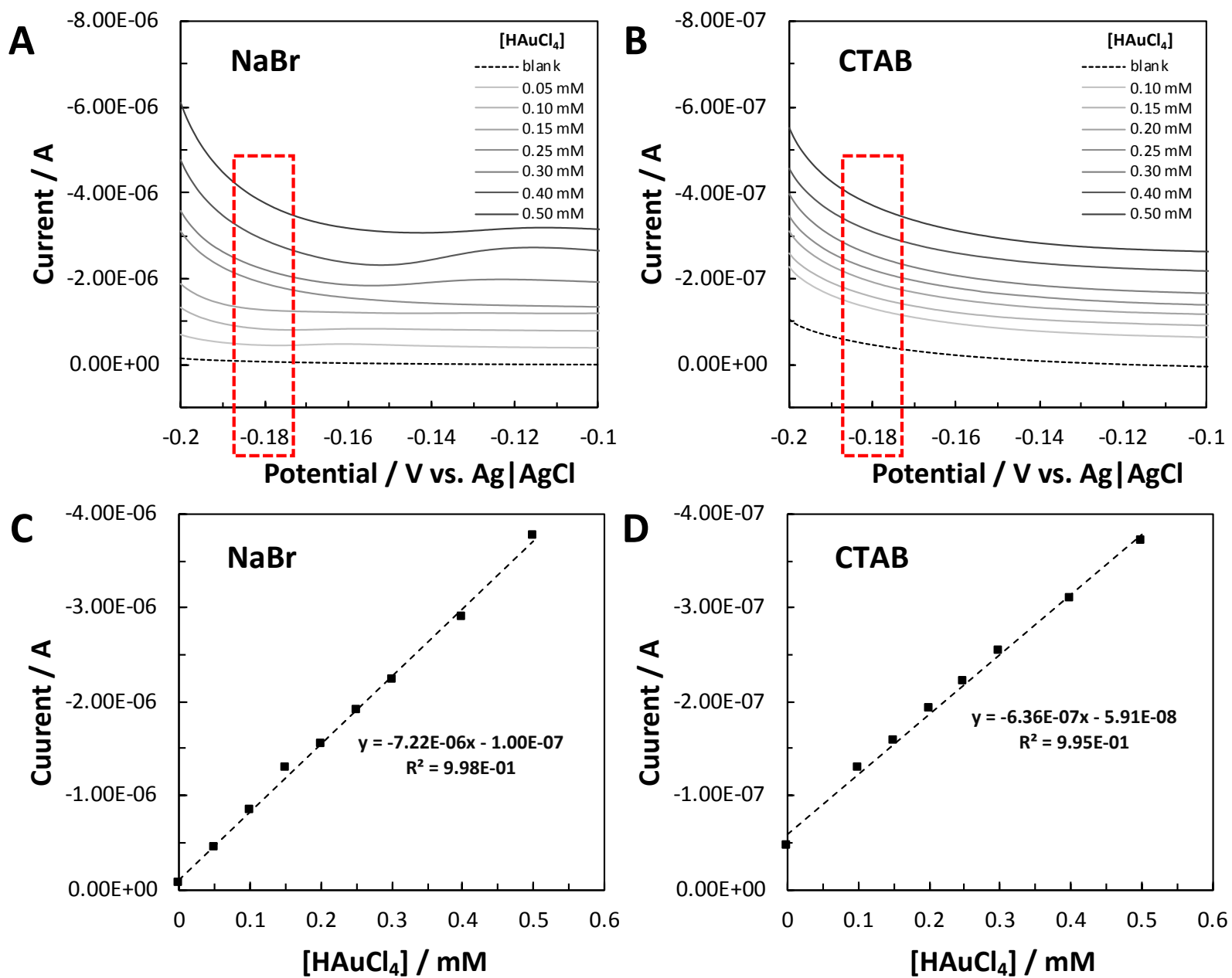

Figure S5. Sequential linear sweep voltammograms collected on polycrystalline Au electrodes in solutions of increasing $\mathrm{HAuCl}_{4}$ concentration, $0.2 \mathrm{M} \mathrm{NaF}$, and either (A) $50 \mathrm{mM} \mathrm{NaBr}$ or (B) 50 $\mathrm{mM}$ CTAB. The dashed line corresponds to a background trace collected in the absence of $\mathrm{HAuCl}_{4}$. The red dashed box indicates the set of current points (arbitrarily selected at $-0.18 \mathrm{~V}$ vs. $\mathrm{Ag} \mid \mathrm{AgCl})$ used to generate the calibration curves shown in (C) and (D) for each set of solutions, respectively. 

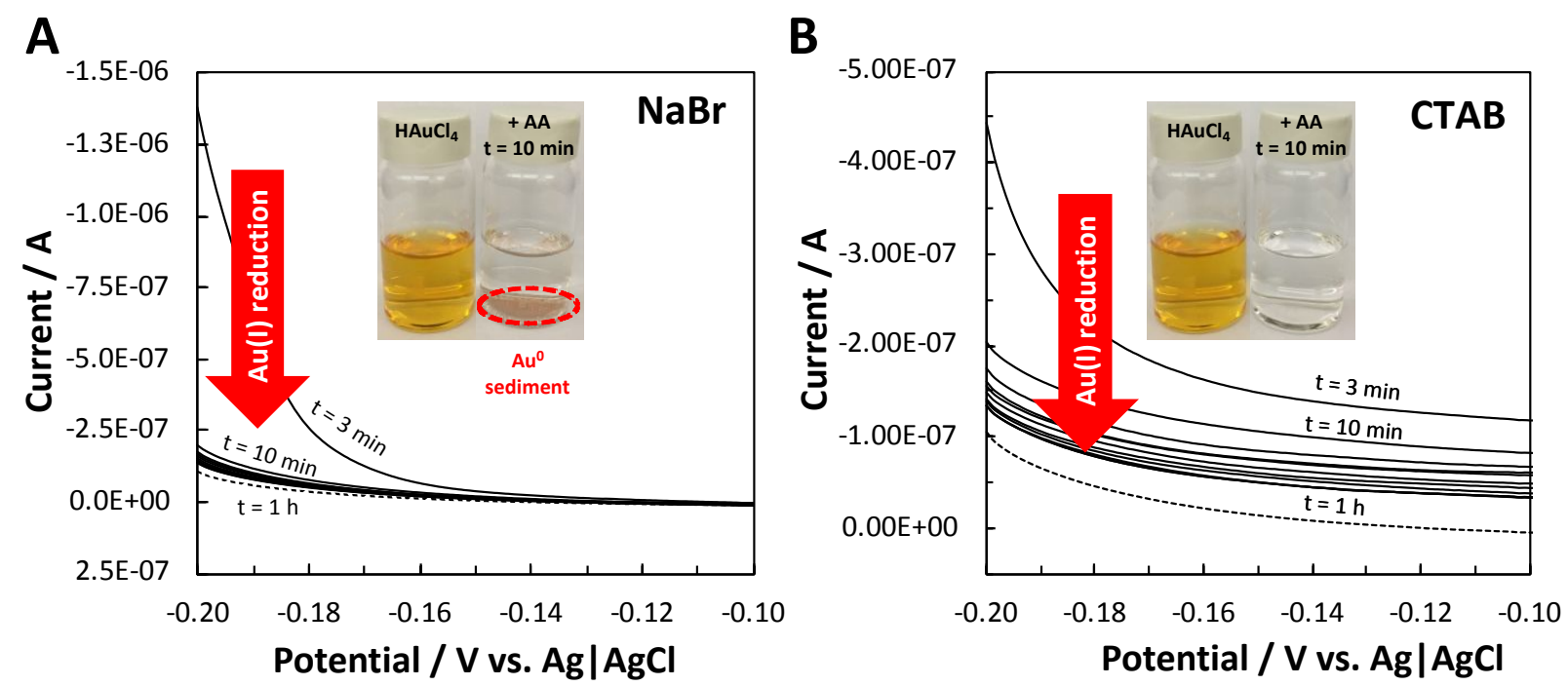

Figure S6. Sequential linear sweep voltammograms collected on polycrystalline Au electrodes in $10 \mathrm{~mL}$ seedless growth solutions containing $0.2 \mathrm{M} \mathrm{NaF}, 0.25 \mathrm{mM} \mathrm{HAuCl}_{4}$, and either (A) $\mathrm{NaBr}$ or (B) $50 \mathrm{mM} \mathrm{CTAB}$. Timepoints reference the point of injection of $55 \mu \mathrm{L}$ of $100 \mathrm{mM}$ AA and refer to the first, second, and tenth traces collected. The dashed line corresponds to a background trace collected in the absence of $\mathrm{HAuCl}_{4}$. Inset photographs depict the respective growth solutions before (yellow-orange) and 10 min after (clear) injection of AA. 


\section{Supplemental tables}

Table S1: Gold nanorod dimensions with increasing NaBr concentration.

\begin{tabular}{|c|c|c|c|c|c|c|}
\hline $\begin{array}{c}{[\mathbf{N a B r}]^{a}} \\
(\mathbf{m M} \mathbf{M})\end{array}$ & $\begin{array}{c}\text { Length }^{b} \\
\text { (nm) }\end{array}$ & $\begin{array}{c}\text { Width }^{b} \\
\text { (nm) }\end{array}$ & $\begin{array}{c}\text { Aspect } \\
\text { Ratio }^{c}\end{array}$ & $\begin{array}{c}\text { Averaged } \\
\text { Aspect } \\
\text { Ratio }^{d} \\
\end{array}$ & $n$ & $\begin{array}{l}\text { Nanorod } \\
\% \text { Yield }^{e}\end{array}$ \\
\hline 0 & $38 \pm 9$ & $\mathrm{n} / \mathrm{a}$ & $\mathrm{n} / \mathrm{a}$ & $\mathrm{n} / \mathrm{a}$ & 2364 & 0 \\
\hline 6.25 & $97 \pm 12$ & $41 \pm 4$ & $2.4 \pm 0.4$ & $2.3 \pm 0.4$ & 319 & 33.3 \\
\hline 12.50 & $108 \pm 20$ & $40 \pm 5$ & $2.7 \pm 0.6$ & $2.7 \pm 0.6$ & 138 & 21.3 \\
\hline 25.00 & $116 \pm 24$ & $30 \pm 3$ & $4.0 \pm 0.9$ & $3.9 \pm 0.9$ & 295 & 22.2 \\
\hline 50.00 & $265 \pm 34$ & $21 \pm 2$ & $11.7 \pm 2.4$ & $12.5 \pm 1.9$ & 410 & 31.9 \\
\hline 62.50 & $281 \pm 37$ & $18 \pm 1$ & $16.0 \pm 2.0$ & $15.8 \pm 2.3$ & 69 & 12.7 \\
\hline 75.00 & $349 \pm 40$ & $18 \pm 1$ & $19.5 \pm 2.4$ & $19.5 \pm 2.4$ & 653 & 43.0 \\
\hline 100.0 & $305 \pm 112$ & $22 \pm 4$ & $14.8 \pm 6.4$ & $13.9 \pm 5.9$ & 447 & 18.6 \\
\hline 125.0 & $322 \pm 116$ & $22 \pm 4$ & $15.1 \pm 6.1$ & $14.5 \pm 5.7$ & 370 & 25.9 \\
\hline
\end{tabular}

${ }^{a}$ Added to growth solutions each containing $50 \mathrm{mM} \mathrm{CTAC}, 0.55 \mathrm{mM} \mathrm{AA}$, and $0.25 \mathrm{mM} \mathrm{HAuCl} 4 .{ }^{b}$ Measured using Image J and SEM micrographs. Measurements include only nanorods with the exception of the $0 \mathrm{mM} \mathrm{NaBr}$ synthesis. ${ }^{c}$ Average of each nanorod's individual aspect ratio. ${ }^{d}$ Calculated from average nanorod lengths and widths. ${ }^{e}$ Calculated from counts of nanorods $(n)$ divided by the total number of distinct nanoparticles in SEM micrographs. Micrographs were collected after particle separation, and thus yields are not representative of synthesis yields. 
Table S2: Iodide content of reagents as measured by ICP-MS.

\begin{tabular}{|c|c|c|c|c|c|}
\hline Reagent & Form & Supplier & $\begin{array}{l}\text { Reported } \\
\text { Purity }\end{array}$ & $\begin{array}{c}{\left[\mathbf{I}^{-}\right]} \\
(\mathbf{p p b})^{a}\end{array}$ & $\begin{array}{l}{\left[\mathbf{I}^{-}\right] \text {in solution }} \\
(\mathbf{n M})^{b}\end{array}$ \\
\hline CTAB & crystalline & Sigma-Aldrich & $\geq 99 \%$ & $116 \pm 15$ & $16.7 \pm 2.1$ \\
\hline CTAC & 25 wt. $\%$ in $\mathrm{H}_{2} \mathrm{O}$ & Sigma-Aldrich & $\geq 99 \%$ & $45 \pm 13$ & $1.4 \pm 0.4$ \\
\hline $\mathrm{NaBr}$ & crystalline & Sigma-Aldrich & $\geq 99.5 \%$ & $74 \pm 9$ & $3.0 \pm 0.4$ \\
\hline
\end{tabular}




\section{References}

1. Kim, M. J.; Brown, M.; Wiley, B. J., Electrochemical investigations of metal nanostructure growth with single crystals. Nanoscale 2019, 11 (45), 21709-21723. 